\title{
WILEY-VCH
}

DOI: $10.1002 /$ ((please add manuscript number))

Article type: Full Paper

Title:

\section{Spectral tuning of high order plasmonic resonances in multimodal film-coupled crystalline cavities}

Upkar Kumar, Aurélien Cuche, Jadab Sharma, Antoine Moreau, Gérard Colas des Francs, Christian Girard, Erik Dujardin

Dr. U. Kumar, Dr. A. Cuche, Dr. C. Girard, Dr. E. Dujardin

CEMES, University of Toulouse and CNRS (UPR8011),

29 rue Jeanne Marvig, BP 94347, 31055 Toulouse, France

E-mail: aurelien.cuche@cemes.fr

Dr. J. Sharma

CNSNT, Panjab University, Chandigarh, India

Dr. A. Moreau

Clermont University, University of Clermont Auvergne,

Pascal Institute, BP 10448, F-63000 Clermont-Ferrand, France

Dr G. Colas des Francs

ICB, UMR 6303 CNRS, Bourgogne Franche-Comté University,

9 Avenue Savary, BP 47870, 21078 Dijon Cedex, France

Keywords: Dark-field spectroscopy, plasmon, nano-optics, MIM nanocavity, high-order resonances

Abstract: Sub-micrometric and ultrathin gold cavities sustain several high order planar plasmon resonances in the visible to near infrared spectral window that open new perspectives for the realization of self-assembled metasurfaces or integrated components for nano-optics. This article investigates in detail the far-field spectral features of these multimodal crystalline gold nanoprisms, deposited on either dielectric (glass) or metallic substrates ( $\mathrm{Au}, \mathrm{Al})$ by darkfield scattering spectroscopy. Relying on the computation of the plasmonic density of states, the signature of each planar resonance is de-convoluted from the experimental global response of single cavities as a function of the cavity size and the substrate nature. The red-shifting dispersion of each resonance is extracted from this decomposition analysis and agrees with predictions from a Green Dyadic Method based numerical tool. In addition, in the presence of 


\section{WILEY-VCH}

a metallic film, we observe a characteristic red or blue shift of the global response for each cavity that results from a metal-specific redistribution of the resonances. The fine spectral tuning of high order plasmonic resonances achieved here reveals the potential of the metalinsulator-metal gap geometry for a static modal engineering within ultrathin gold cavities.

\section{Introduction}

Metallic nanoparticles have been at the focus of numerous studies owing to their ability to sustain plasmonic resonances in the visible, which make possible the control of light at the nanoscale [1]. Plasmonic resonances in metallic nanostructures allow for a strong confinement and enhancement of the electromagnetic field in subwavelength volumes along with plasmon propagation in structures with low dimensionalities [2]. These appealing properties have triggered the development of biosensing [3], energy conversion [4,5], detectors [6], integrated optical component for information transfer and/or processing [7], or shaping of the phase front of light $[8,9]$. Several applications rely on the ability of the plasmonic nanoparticle to efficiently scatter or concentrate the light in a specific region of space at a given energy. As a consequence, a constant effort has been undertaken to control and tune the plasmon resonance of subwavelength plasmonic resonators or antennas from UV to IR [10,11]. Strategies to adjust the conditions of resonance or propagation rely on the static $[12,13]$ or dynamic $[14,15,16]$ modification of the surrounding medium, on the modification of the geometry [17], the size or the material of the metallic object itself $[12,18,19]$, or on the modification of the charge carrier density, for instance [20].

A particularly efficient approach exploits the electromagnetic coupling between a plasmonic nanoparticle and a thin metallic film [21-26]. The small nanocavity resulting from the metalinsulator-metal (MIM) geometry gives rise to new longitudinal and transverse plasmonic resonances in the gap along with a spectral shift of the native resonance of the nanoparticle [27- 


\section{WILEY-VCH}

29]. The adjustment of the coupling strength by varying the distance between nanoparticles and the conductive plasmonic film can be used to tune the SP resonance wavelength [30-32]. The MIM geometry, which is compatible with operational device configuration, offers therefore a new degree of freedom to control the modal plasmonic landscape and the related scattering properties of the system.

While the optical response of subwavelength particles is well defined and usually limited to one or two resonances in the visible, sub-micronic two-dimensional (2D) colloidal gold cavities sustain multiple higher order plasmonic resonances that offer a much richer multimodal plasmonic spectrum. Indeed, these highly crystalline and thin metallic cavities exhibit a single transverse mode but several planar resonances of higher order than the dipolar one in the visible and near-infrared [33-36]. They result from multiple interferences between degenerated highorder SP modes and yield optical near-fields that are strongly localized at specific hot spots along the edges and at the center of the structures. As a consequence, these high order resonances combine the advantages of localized field enhancement and delocalized 2D remote connection between these electric field hotspots. These versatile optical properties of ultra-thin plasmonic cavities position them as promising building blocks for the development of 2D nanophotonic components for optical information processing and transfer [34]. Such development of integrated components with clearly defined functionalities requires an ondemand spatial and spectral tailoring of the plasmonic resonances in the system. This concept has recently been demonstrated for the control of the optical signal transmission through the design of plasmon eigenstates [37]. While weak electromagnetic coupling between two cavities has been discussed as promising approaches [38,39], including the coupling between a cavity and a resonant defect, the MIM geometry therefore appears as a more effective and more tunable configuration for an efficient spectral modal engineering and actuation in these 2D systems. 


\section{WILEY-VCH}

In the present work, we perform a comprehensive study of the far-field scattering response of individual crystalline Au prismatic cavities of different sizes ranging from 400nm to $900 \mathrm{~nm}$ as a function of the substrate. We first record the forward scattering spectra of these objects on a dielectric substrate by dark-field (DF) scattering spectroscopy. Then, we decompose the global response into a series of red-shifting longitudinal Lorentzian resonances thanks to a qualitative comparison with the planar near-field plasmonic local density of states (SP-LDOS) simulated with a Green Dyadic Method based numerical tool. We extend our analysis by examining the spectral behavior of these $\mathrm{Au}$ cavities deposited on 30nm thin metallic films in a MIM configuration. When the distance between the nanoprisms and the thin films is kept constant, but the metal is changed from Au to Al, we observe a characteristic global blue shift, confirming the potential of the MIM nanocavity configuration for an on-demand static spectral modal engineering.

\section{Results and discussion}

\subsection{High order plasmonic resonances in crystalline cavities}

Earlier studies on the near-field optical properties of such objects have revealed the spatial distribution of the high order resonances occurring in the plane of the cavity. These works mainly relied on non-linear photoluminescence microscopy [34-36] or electron probes [33]. Both approaches are based on the total absorption or the partial loss of energy in a plasmonic channel. The signal measured is therefore proportional to the SP-LDOS in the cavity, which provides a local and near-field information $[34,40]$. Yet, the coupling of these modes to the far field has not been discussed in detail. When illuminating a single object in the visible, several resonances are probed together, it is therefore difficult to infer the contribution of each of them in the intertwined far-field signal. DF scattering spectroscopy is an all-optical technique that 


\section{WILEY-VCH}

measures the purely scattered optical signal associated with SP resonances in individual metallic nanostructures (Figure 1). This simple and robust microscopy has already been used for the characterization of MIM nanocavity geometries [24,25].

Figure 1(a) shows the DF spectrum recorded on a gold cavity with lateral size of $460 \mathrm{~nm}$. The forward scattering intensity exhibits the presence of two dominant overlapping peaks and a low intensity plateau at longer wavelengths in a spectral window ranging from $475 \mathrm{~nm}$ to $825 \mathrm{~nm}$. This spectrum cannot accommodate a single resonance as observed in small dipolar spherical particles at similar energies. Assuming that SP resonances can be described as damped harmonic oscillators driven by the incident electric component of light [41], we propose a decomposition method based on the summation of Lorentzian functions to fit the experimental scattering spectra. This approach allows the extraction of the characteristic parameters of the individual resonance peaks potentially associated to the high order SP resonances in the cavities, as illustrated by the four colored Lorentzian curves in Figure 1.

The forward DF spectra recorded on single gold cavities with different sizes (a subset of examples is shown in Figure 1(b)) were systematically fitted with multiple Lorentzian peaks [41]. This fitting was done by an iterative sequence until a minimal residue is reached. However, the parameter space defining the fitting method (peak position, peak number, peak width and area) is large for such 2D systems, leading to multiple equivalent and valid fitting solutions. This convergence issue can be settled by making specific hypotheses on several parameters related to plasmonic resonances. First, the full width at half maximum (FWHM) of a SP resonance is mainly determined by the damping of the SP oscillations. This damping is driven by two components related to internal and radiative losses [42,43]. Given the large dimensions of the gold cavities, and their rather low symmetry, our first assumption is to consider all these high order resonances bright with an equal radiative damping. The internal contribution to 


\section{WILEY-VCH}

losses is related to the amplitude of imaginary part of the dielectric function $\operatorname{Im}(\varepsilon)$. The latter includes the different contributions involved in the dephasing process in metal nanoparticles like electron scattering into empty levels of conduction bands and electron - phonon coupling. It also accounts for the interband transitions that mainly occurs for wavelengths below $600 \mathrm{~nm}$ in gold. The evolution of $\operatorname{Im}(\varepsilon)$ in the $500-850 \mathrm{~nm}$ spectral window $(\Delta \omega)$ led us to impose a decreasing of the FWHM of each peak of a given structure as the peak's central wavelength increases.

The second constraint has been set on the number of fitting Lorentzian curves, $N$, and their initial spectral position, $\lambda_{N}$. For subwavelength nanoparticles, the few resonances occurring in the visible can be efficiently numbered and separated. It is therefore convenient to compare the experimental data to the simulated scattering cross-section. For larger plasmonic systems, the computation of the scattering cross-section fails to reproduce the experimental data as shown in Figure 2(a). These discrepancies mainly originate from the size of the platelets, and a more refined and complex approach is needed [44]. Moreover, in the present case of experimental spectra with multiple overlapping peaks (see Figure 1), the simulation of the global response (scattering cross-section) provides limited information on each single resonance.

In order to overcome these difficulties, we apply an original method to infer the values of $N$ and $\lambda_{N}$. We systematically and qualitatively compare the experimental scattering spectra with simulated planar SP local density of states (SP-LDOS) $\rho_{\|}(r, \omega)$ computed on the same spectral range $\Delta \omega$ with the Green Dyadic method.

$$
\rho_{\|}(\boldsymbol{r}, \omega)=\frac{1}{2 \pi^{2} \omega} \operatorname{Im}\left\{S_{x, x}(\boldsymbol{r}, \boldsymbol{r}, \omega)+S_{y, y}(\boldsymbol{r}, \boldsymbol{r}, \omega)\right\}
$$

with $\boldsymbol{r}=(x, y, z)$ an arbitrary position in the metal and $\omega$ the angular frequency. $S_{x, x}(\boldsymbol{r}, \boldsymbol{r}, \omega)$ and $S_{y, y}(\boldsymbol{r}, \boldsymbol{r}, \omega)$ are the first two diagonal components of the Green dyadic tensor $S(\boldsymbol{r}, \boldsymbol{r}, \omega)$ calculated at the position $\boldsymbol{r}$ inside the metallic structure. The planar SP-LDOS describes all the 


\section{WILEY-VCH}

available planar states in the cavity at a given position and energy, independently of the incident illumination. Once $N$ and $\lambda_{N}$ have been estimated by this numerical analysis, they are incorporated as a fixed parameter, for $N$, and starting values, for the peak positions $\lambda_{N}$. The iterative fitting sequence is run with the peak positions $\lambda_{N}$ left free to evolve until a converged fit to the full spectrum is obtained which fulfill the first constrain (FWHM evolution).

Figure 2(a-d) presents the DF spectra of four different Au cavities with lateral sizes of 430, 565, 650 and $710 \mathrm{~nm}$ respectively. Each DF spectrum is presented with the total fit (orange curve) and the corresponding constituting Lorentzian peaks. Interestingly, the overall scattering response of these four objects, with noticeable size differences, occurs in the same spectral window, with only slight modulations of this broad peak as a function of the wavelength. The good agreement between the experimental curves and the total fits shows that our fitting approach based on a limited number of Lorentzian peaks is satisfactory. In the lower inset of Figure 2(a), we show the weak contribution of the transverse mode (along the thickness of the platelet). It is systematically dominated by the signal from the planar resonances which justifies to only compute the planar density of states. Along with experimental data, the corresponding simulated near-field SPLDOS spectra are displayed in Figure 2(e-f). For each object, the mesh for the simulation is adjusted to match the experimental geometry as closely as possible. As stated in Equation 1, the planar SP-LDOS is computed at a given position in the metal and therefore provides a near-field and local information. The spatial distribution of the high order resonances exhibits nodes and antinodes along the edge of the cavities as demonstrated in former works on similar prismatic systems $[33,38,39]$. Accordingly, we compute the planar SPLDOS in two specific positions, namely the corner and the middle of an edge (see inset of Figure 2(e)), guarantying to probe the whole spectral plasmonic landscape of the cavities [38]. The SP-LDOS spectra in Figure 2 show that the global shape of the SP-LDOS, each displaying several peaks, and the DF experimental spectra lie in the same spectral range. In addition, for a given size, the converged fit shows that the position of the individual Lorentzian peaks remains 


\section{WILEY-VCH}

consistent with respect to the SP-LDOS ones. Dashed black lines, aligned with the SP-LDOS peaks, have been drawn on Figure 2 to emphasize this correspondence.

A direct quantitative comparison of the simulated planar SP-LDOS spectra with the experimental DF scattering spectra should not be established because the former provides a local and near-field information in the metal, with no clue on the far-field coupling, while the latter is a spatially integrated measurement in the far field. In particular, a redshift between near- and far-field spectra is expected. Yet, a qualitative comparison suggests that several planar high order SP resonances are contributing to the forward light scattered by these plasmonic systems, justifying the use of SP-LDOS spectra as guidelines for fitting.

Interestingly, the comparison between the Lorentzian decomposition and the SPLDOS analysis can be used beyond a simple guiding methodology. By computing the spatial distribution map corresponding to each SP-LDOS peak, the order of each resonance is inferred from the number of antinodes displayed. As shown in the insets of Figure 2(e-h), every single peak or shoulder in the spectra originates from a different resonance order with a singular SP-LDOS spatial distribution. All of them are characterized by an incremental number of antinodes and/or an incremental number of maxima at the central core of the prism as the energy increases. These resonances can thus be easily followed from one cavity size to the next, drawing a clear redshift of these high order planar SP resonances as the cavity size increases. This observation is in good agreement with an earlier work on the same gold system where, for a fixed wavelength, an increase of the triangular cavity size leads to the build-up of a new SP resonance of higher order [35,37]. Thanks to the consistent relationship between fitted and simulated resonances, the Lorentzian peaks are correlated from one spectrum to the other, and a resonance order is attributed through a color code as shown in Figure 2. It appears clearly that each single experimental resonance is red shifting as the cavity size increases, with new resonances emerging in the high energy part of the spectrum. Our observations confirm that a threshold 


\section{WILEY-VCH}

value of the edge length increase (about $100 \mathrm{~nm}$ ) is necessary to accommodate a new resonance in the fit of the experimental curve (see Figure 2(e), (f) and (g)). For instance, between Figures $\mathbf{2}(\mathrm{g})$ and $\mathbf{2}(\mathrm{h})$, the difference in size is not sufficient and the same resonances are observed with a significant red shift.

Applying the same assignment to the full set of fitted and simulated data, a high order SP resonance dispersion can be constructed. These data are gathered in Figure 3(a) and (b) where a similar trend is observed for all the resonances in both fitted and computed spectra. The resonances are associated to Fabry-Perot behavior of the plasmonic cavity [45]. A possible improvement of our approach would be the extraction of the wavevectors (k) associated to the plasmon resonances in the SP-LDOS maps, and their subsequent dispersion relation $(\mathrm{k}(\omega))$.

The full set of SP-LDOS spatial distributions associated to the resonances available in these prismatic cavities is presented in Figure 3(c). These resonances display a quality factor between 7 and 15, which is in good agreement with the values reported in the literature [41]. This observation relates to our assumption of an equal radiative damping for these high order planar resonances. These computed spatial distributions are in excellent agreement with the ones described as edge (from light blue to pink) and breathing eigenmodes (dark red and dark green) in similar prismatic geometries $[33,45,46]$. The experimental peak decomposition provides an in-depth description of the set of plasmonic resonances sustained by the platelets. It also gives precious indications on their coupling to the far field, since the signature of all the resonances identified in the SPLDOS spectra is recorded in the far field, meaning that even the high order contributions have a radiative behavior. The detailed spatial and spectral mapping of the resonances as a function of the cavity size found here is a useful guide for the targeted alteration of one specific subset of resonances, for example by local etching as shown in [38], and thus a rational engineering of the plasmon modes in these crystalline nanoprisms [37].

\subsection{Spectral control of high order SP resonances in a MIM configuration}




\section{WILEY-VCH}

The same colloidal suspension of Au nanoprisms is deposited on a semi-transparent (thickness of $30 \mathrm{~nm}$ ) sputtered gold film with a spacing layer of approximately $3 \mathrm{~nm}$. The spacer corresponds to a residue of a cross-linked polymer that is not removed during the cleaning steps and is always present below the colloid as discussed in a previous work (see methods).

The MIM configuration has already demonstrated its potential for the spectral tuning of dipolar and quadrupolar resonances in subwavelength metallic particle $[25,26]$. Figure 4(a) and (d) show two DF images recorded on the two different substrates. The DF image on the ITOcovered glass substrate shown in Figure 4(a) reveals an orange-red tinted scattering of the colloidal cavities while the forward scattering of these objects recorded on the $\mathrm{Au}$ thin film appears to be shifted in the yellow and green parts of the spectrum. This trend is quantitatively confirmed by a systematic acquisition of scattering spectra from sixteen prismatic cavities on gold with similar sizes to the objects discussed in the first section. A comparison between the two systems is shown in Figure 4 for two different cavity sizes, namely $450 \mathrm{~nm}$ in (b) and (e), and $650 \mathrm{~nm}$ in (c) and (f). For a given size, the total spectrum undergoes a blue shift of about $50 \mathrm{~nm}$ in all cases, demonstrating the strong effect of the metallic film on the optical response of the platelets and the potential of the MIM nanocavity geometry for modal control.

From the comparison of the spectra in Figure 4 recorded either on glass or gold, it is difficult to infer if the global blue shift of the whole spectra originates from the blue shift of each individual Lorentzian components or whether significant intensity redistribution occurs, possibly alongside a spectral shift. A spectral blue or red shift of the plasmon resonance can be induced by the static injection of charges in the resonant system [20,47]. But the charge transfer by a tunneling mechanism is unlikely considering the rather thick spacing layer and the absence of applied bias on the metal film. Blue shift of plasmon modes has already been reported in $\mathrm{Au}$ nanorods deposited on Au thin film with respect to one deposited on glass in a previous work 


\section{WILEY-VCH}

[48]. In that case, the electromagnetic coupling between the particle and the film leads to the spectral shift. Depending on the strength of this interaction, which is experimentally done by changing the thickness of the spacer, the scattering spectra can undergo blue or red shifts. In these studies, the subwavelength size of the nanorod made possible an easy tracking of the spectral evolution of the longitudinal and transverse resonances.

In order to validate our numerical approach in a MIM configuration and to test its accuracy, we first compute the SP-LDOS spectra in a $60 \mathrm{~nm}$ gold nanosphere positioned $15 \mathrm{~nm}$ above either a glass substrate or a $45 \mathrm{~nm}$ gold film, as a benchmark, following the geometry of reference 31 . The plasmonic response of the sphere is presented in Figure 5(a). For such isolated plasmonic resonances, a clear red shift is observed as discussed in the literature for similar systems [25]. To circumvent the problem of overlapping resonances, we applied again our SP-LDOS-assisted methodology for the fitting of the experimental DF spectra on gold. We limited the numerical investigation to the four smaller prims because of the dramatic increase of the generalized propagator computing time, which now accounts for the presence of the infinite $2 \mathrm{D}$ metallic film.

Figure 5(b) shows the dispersion of the resonances identified in the cavities in the MIM geometry. After computation of the spatial distributions for each peak identified in the SPLDOS spectra, we applied the same color code as Figure $\mathbf{3}$ ( for cavity-on-glass configuration) to the dispersion curves in Figure 5(b) for MIM geometry. This numerical experiment confirms that every single high order planar resonance is redshifted compared to the reference on glass. Next, we use a simple linear fit (colored dashed lines in Figure 5(b)) to extrapolate the spectral positions of these identified resonances for the larger cavity sizes in order to facilitate the fitting of the experimental spectra.

For this gold MIM geometry, we observed a systematic 50-100 nm redshift of the global maximum position of the simulated spectra with respect to the experimental one that exhibit a global maxima around 600-650 nm. Below $650 \mathrm{~nm}$, it has been more difficult to clearly identify 


\section{WILEY-VCH}

the resonances. Along with time computing constrain, a set of cavity size - wavelength configurations has consequently not been explored. It leads to a shaded area in the lower half of the graphics in Figures 5(b) and (c) where the position of the resonances in the SP-LDOS cannot be accurately defined. Nevertheless, the assessment of the first four resonances position allowed us to fix a value for $\lambda_{N}$. We then applied the iterative fitting sequence. A dispersion graph can be reconstructed for each fitted Lorentzian curve with the same aforementioned color code. These results are presented in Figure 5(c). A similar evolution is observed for the four first resonances between SP-LDOS and fitted peaks. It is worth noting that the Lorentzian peak positions can be linked in the same way in the shaded area of the graph. Although not related to SP-LDOS maxima, these Lorentzian peaks draw resonances with the same slope as the colored ones. It can be reasonably attributed to high order planar resonances building up in the cavity. Our analysis undoubtedly reveals a redshift of every single planar resonance of the cavity in the MIM configuration, which is counterintuitive regarding the blue shift of the global spectra. An increase of the quality factor associated to the first order modes of a spherical nanoparticle has been observed in a MIM geometry by reduction of the radiative damping [49]. This electromagnetic coupling, depicted as a hybridization mechanism between the modes of the particle mediated by the underlying film, leads to a subsequent narrowing of the resonances. In our system, the Lorentzian fitted peaks associated to the high order resonances of the cavity do not show a significant modification of the quality factor in comparison to the particle-onglass situation. Over the sixteen sizes explored, the quality factors extracted from the fits range from 7 to 14 . These values are statistically equivalent to the ones obtained on glass and remain low.

However, this interpretation of the physical origin of the fitted peaks does not account for the contributions of plasmonic gap modes. For instance, in film-coupled nanoparticles, strong cavity resonances due to the excitation of a gap-plasmon along the cavity and film faces are expected [28]. 


\section{WILEY-VCH}

We therefore solved the dispersion relation of the structure using a complementary numerical tool optimized for the modelling of wave propagation in multilayer systems [50]. We present on Figure 6 the computed profiles of the real part of the magnetic field for the modes supported by both configurations (prism on glass in Figure 6(a) and on gold in Figure 6(b) and (c)). Computing the wavevector of the different modes (and the effective index $n=k_{x} / k_{0}$ ) allows to unambiguously identify which mode is responsible for the resonances presented in Figure 3. For a lateral size of the prism of $400 \mathrm{~nm}$ and a wavelength of $600 \mathrm{~nm}$, the effective index around 1.02 of the upper surface plasmon, shown Figure 6(a), is the only on compatible with the number and characteristics of the resonances. We can conclude that only the upper bound high order plasmonic resonance is probed in our study, which is consistent with the discussion in section 2.1. Interestingly, the gap-plasmon resonance (blue profile in Figure 6(c)) presents a wavevector (with an effective index of $\operatorname{Re}\left(\mathrm{n}_{\mathrm{gp}}\right)=11.46$ ) which is tremendously higher than the wavevector of the incoming photons. In addition, the imaginary part of the effective index is also high with a value of 0.82 (propagation length of $120 \mathrm{~nm}$ )- which has to be compared to the value of the upper bound plasmon of 1.02 (propagation length of $\sim 5 \mu \mathrm{m}$ ). Such a strong damping, correlated to the momentum mismatch, prevents the excitation of the longitudinal gap plasmon at these wavelengths [51]. Consequently, the gap resonance does not contribute to the far-field signal.

Our results indicate that the MIM geometry is able to spectrally shift the high order resonances of these ultra-thin cavities. One possibility to control the amplitude of the shift is by adjusting the thickness of the insulator layer that tunes the strength of the film-particle coupling. Alternatively, keeping the polymer spacer constant, we vary the nature of the metal and its associated plasmonic response. DF scattering spectra are recorded from Au cavities deposited on a substrate coated with a layer of $30 \mathrm{~nm}$ of Aluminum. The normalized DF spectra for three 


\section{WILEY-VCH}

cavities of sizes 450nm, 600nm and 725nm are presented in Figure 7(a), (b) and (c) on two different substrates - Al (blue), Au (black) - respectively. The plots show a systematic blue shift of the global scattering response when the gold film is replaced by aluminum. A similar blue shift has also been predicted for silver nanocubes deposited on thin gold and silver films [28]. The modal decomposition allowed by the SP-LDOS analysis reveals that, unlike the Au-Au MIM configuration, not only the global DF response is blue shifted, but also every single high order resonance. The shift of two of these resonances, extracted from the SP-LDOS spectra, are displayed in Figure 7(d). It shows a blueshift of merely $60 \mathrm{~nm}$ when an Al film is used instead of $\mathrm{Au}$. With these results, we demonstrate that the choice of the metal below the gold cavities is an efficient mean for tuning the resonant conditions of the cavity in the MIM configuration, which makes possible an on-demand spectral tuning of the high order planar resonance at work in these cavities.

This concept could be pushed further by acting on the other degrees of freedom of the MIM system (gap width and refractive index, etc) $[52,53]$. Yet, such exhaustive study is beyond the scope of this work.

\section{Conclusion}

In conclusion, the detailed forward scattering response of planar gold prismatic cavities with sizes ranging from 400 to $900 \mathrm{~nm}$ have been systematically investigated by dark-field scattering spectroscopy. The global dark-field spectra were decomposed by multipeaks Lorentzian fitting thanks to a qualitative comparison with near-field SPLDOS spectra. This analysis demonstrated that each planar mode observed in the simulation can be bound to a single peak in the intertwined experimental spectra, and experienced a redshift as the cavity size increases. Our all-optical approach allows the reconstruction of the full plasmonic landscape for these planar resonances. 


\section{WILEY-VCH}

By changing the dielectric substrate for a metallic film ( $\mathrm{Au}$ or $\mathrm{Al}$ ), in a MIM configuration, we demonstrate that a red- or blue-shift of the global and/or single resonances is obtained. This strategy allows for an efficient modal engineering in these cavities in a MIM geometry. Our work opens the door to the spectral control of the plasmonic landscape associated to these $2 \mathrm{D}$ cavities, which might find some application in the design of plasmonic planar components and metasurfaces [54,55].

\section{Experimental Section}

A suspension of crystalline Au nanoprisms was obtained by reduction of Au precursors by polyvinylpyrrolidone (PVP) by a one-pot method at room temperature. These nanoprisms are $20 \mathrm{~nm} \pm 3$ in thickness and with lateral dimensions ranging from $300 \mathrm{~nm}$ to $1.5 \mu \mathrm{m}$ [34]. This suspension is then drop casted and dried onto $10 \mathrm{~nm}$ ITO coated glass substrate to obtain $\mathrm{Au}$ nanoprisms with high aspect ratio. The nanoprisms are then subjected to 5 min oxygen plasma to remove PVP from the surfaces. While the upper surface is thoroughly cleaned from all organic adsorbates, the $\mathrm{O}_{2}$ plasma does not fully remove the PVP at the interface between the nanoprims and the substrate. In the gap, PVP is partially cross-linked leading to a ca. 3-nm thick, organic-filled spacing. [56]. The single particle dark-field scattering spectroscopy was performed using a custom-built dark field spectroscopy set up where a halogen white light source was focused using a Nikon dry dark-field condenser (NA: 0.8-0.95) onto the sample (see Figure 1(a)). Scattered light was collected in transmission (diascopic) configuration using a 40X Nikon objective with NA: 0.72 . The light was focused on to conjugate image plane where a pinhole $(50 \mu \mathrm{m})$ is placed to select scattering from a single nanoprisms (it corresponds to a collection area with a diameter of $1.25 \mu \mathrm{m}$ around the selected object) and to remove unwanted scattering from nearby adjacent nanoparticles. The broadband spectral background was removed by subtracting the scattered light from a region of the substrate near the nanoprism 


\section{WILEY-VCH}

from the raw particle spectrum. The final spectrum was corrected from the spectral efficiency of the entire spectrometer by normalization with the scattering spectrum of the halogen lamp. The experimental and fitting errors are discussed in the Supplementary Material document.

Conflict of Interest: The authors have no financial/commercial Conflict of Interest.

\section{Acknowledgements}

This work was supported by the Agence Nationale de la Recherche (ANR) (Grant ANR-13BS10-0007-PlaCoRe), and the massively parallel computing center CALMIP in Toulouse. We thank Christian Bourgerette for technical assistace.

Received: ((will be filled in by the editorial staff))

Revised: ((will be filled in by the editorial staff)) Published online: ((will be filled in by the editorial staff))

\section{References}

[1] C. F. Bohren, D. R. Huffman, Absorption and scattering of light by small particles. (Wiley science paperback series) 1998, 16, 544

[2] W. L. Barnes, A. Dereux, T. W. Ebbesen, Nature (London) 2003, 424, 824

[3] J. Homola, Chem. Rev. 2008, 108, 462

[4] A. O. Govorov, W. Zhang, T. Skenei, H. H. Richardson, J. Lee, N. A. Kotov, Nanoscale Res. Lett. 2006, 1, 84

[5] G. Baffou, R. Quidant, C. Girard, Appl. Phys. Lett. 2009, 94, 153109

[6] G. Li, S.-W. Wang, X. Chen, W. Lu, Appl. Phys. Lett. 2014, 104, 231104

[7] D. K. Gramotnev, S. I. Bozhevolnyi, Nat. Photon. 2010, 4, 83

[8] N. Yu, P. Genevet, M. A. Kats, F; Aieta, J.-P. Tetienne, F. Capasso, Z. Gaburro, Science 2011, 334, 333

[9] G. Li, B. P. Clarke, J-K. So, K. F. MacDonald, N. I. Zheludev, Nat. Comm. 2016, 7, 13705

[10] L. Novotny, N. van Hulst, Nat. Photon. 2011, 5, 83 


\section{WILEY-VCH}

[11] P. Biagioni, J.-S. Huang, B. Hecht, Rep. Prog. Phys. 2012, 75, 024402

[12] K. A. Willets, R. P. Van Duyne, Annual Review of Physical Chemistry 2007 58, 267

[13] S. Link, M. B. Mohamed, M. A. El-Sayed, J. Phys. Chem. B 1999, 103, 3073

[14] P. A. Kossyrev, A. Yin, S. G. Cloutier, D. A. Cardimona, D. Huang, P. M. Alsing, J. M. Xu, Nano Lett. 2005, 5, 1978

[15] J. Kim, H. Son, D. J. Cho, B. Geng, W. Regan, S. Shi, K. Kim, A. Zettl, Y.-R. Shen, F. Wang, Nano Lett. 2012, 12, 5598

[16] M Rudé, R. E. Simpson, R. Quidant, V. Pruneri, J. Renger, ACS Photonics 2015, 2, 669

[17] M. Grzelczak, J. Perez-Juste, P. Mulvaney,L. M. Liz-Marzàn, Chemical Society Reviews 2008, 37,1783

[18] J. J. Mock, M. Barbic, D. R. Smith, D. A. Schultz, S. Schultz, The Journal of Chemical Physics 2002, 116, 6755

[19] B. J. Wiley, S. H. Im, Z.-Y. Li, J. McLellan, A. Siekkinen, Y. Xia, J. Phys. Chem. B 2006, 110,15666

[20] P. Mulvaney, J. Pérez-Juste, M. Giersig, L. M. Liz-Marzàn, C. Pecharromàn, Plasmonics 2006, 1, 61

[21] P. Nordlander, E. Prodan, Nano. Lett. 2004, 4, 2209

[22] F. Le, N. Z. Lwin, J. M. Steele, M. Käll, N. J. Halas, P. Nordlander, Nanolett. 2005, 5, 2009

[23] G. Lévêque, O. J. F. Martin, Optics Lett. 2006, 31, 2750

[24] N. J. Halas, S. Lal, W.-S. Chang, S. Link, P. Nordlander, Chem. Rev. 2011, 111, 3913

[25] T. Maurer, P.-M. Adam, G. Lévêque, Nanophotonics 2015, 4, 363

[26] C. Tserkezis, R. Esteban, D. O. Sigle, J. Mertens, L. O. Herrmann, J. J. Baumberg, J. Aizpurua, Phys. Rev A 2015, 92, 053811

[27] D. Y. Lei, A. I. Fernandez-Dominguez, Y. Sonnefraud, K. Appavoo, R. F. Haglund, Jr., J. B. Pendry, S. A. Maier, ACS Nano 2012, 6,1380

[28] J. Britt Lassiter, F. McGuire, J. J. Mock, C. Ciracì, R. T. Hill, B. J. Wiley, A. Chilkoti, D. R. Smith, Nano Lett. 2013, 13, 5866

[29] Q.-Y. Lin, Z. Li, K. A. Brown, M. N. O’Brien, M. B. Ross, Y. Zhou, S. Butun, P.-C. Chen, G. C. Schatz, V. P. Dravid, K. Aydin, C. A. Mirkin, Nano Lett. 2015, 15, 4699 


\section{WILEY-VCH}

[30] L. He, E. A. Smith, M. J. Natan, C. D. Keating, J. Phys. Chem. B 2004, 108, 10973

[31] J. J. Mock, R. T. Hill, A. Degiron, S. Zauscher, A. Chilkoti, D. R. Smith, Nano Lett. 2008, 8,2245

[32] J. J. Mock, R. T. Hill, Y.-J. Tsai, A. Chilkoti, D. R. Smith, Nano Lett. 2012, 12, 1757

[33] L. Gu, W. Sigle, C. T. Koch, B. Ogut, P. A. van Aken, N. Talebi, R. Vogelgesang, J. L. Mu, X. G. Wen, J. Mao, Phys. Rev. B. 2011, 83, 195433

[34] S. Viarbitskaya, A. Teulle, R. Marty, J. Sharma, C. Girard, A. Arbouet, E. Dujardin, Nat. Mater. 2013, 12, 426

[35] S. Viarbitskaya, A. Teulle, A. Cuche, J. Sharma, C. Girard, E. Dujardin, A. Arbouet, Appl. Phys. Lett. 2013, 103, 131112.

[36] S. Viarbitskaya, A. Cuche, A. Teulle, J. Sharma, C. Girard, A. Arbouet, E. Dujardin, ACS Photonics 2015 2, 744

[37] U. Kumar, S. Viarbitskaya, A. Cuche, C. Girard, S. Bolisetty, R. Mezzenga, G. Colas des Francs, A. Bouhelier, E. Dujardin, ACS Photonics 2018, 5, 2328

[38] A. Cuche, S. Viarbitskaya, J. Sharma, A. Arbouet, C. Girard, E.Dujardin, Sci. Rep. 2015, 5,16635

[39] A. Cuche, S. Viarbitskaya, U. Kumar, J. Sharma, A. Arbouet, C. Girard, E. Dujardin, Opt. Commun. 2017, 387, 48

[40] F. J. Garcia de Abajo, Rev. Mod. Phys., 2010, 82, 209

[41] M. Bosman, E. Ye, S. F. Tan, C. A. Nijhuis, J. K. W. Yang, R. Marty, A. Mlayah, A. Arbouet, C. Girard, M.-Y. Han, Sci. rep. 2013, 3, 1312

[42] C. Sönnichsen, T. Franzl, T. Wilk, G. von Plessen, J. Feldmann, O. Wilson, P. Mulvaney, Phys. Rev. Lett. 2002, 88, 077402-1

[43] M. Hu, C. Novo, A. Funston, H. Wang, H. Staleva, S. Zou, P. Mulvaney, Y. Xiae, G. V. Hartland, J. Mater. Chem. 2008, 18, 1949

[44] L. Jiang, T. Yin, Z. Dong, M. Liao, S. J. Tan, X. M. Goh, D. Allioux, H. Hu, X. Li, J. K. W. Yang, Z. Shen, ACS nano 2015, 9, 10039

[45] A. Campos, A. Arbouet, J. Martin, D. Gérard, J. Proust, J. Plain, M. Kociak, ACS Photonics 2017, 4, 1257

[46] F.-P. Schmidt, H. Ditlbacher, U. Hohenester, A. Hohenau, F. Hofer, J. R. Krenn, Nano Lett. 2012, 12, 5780

[47] C. Goldmann, R. Lazzari, X. Paquez, C. Boissière, F. Ribot, C. Sanchez, C. Chanéac, D. Portehault, ACS Nano 2015, 9, 7572 


\section{WILEY-VCH}

[48] X. Chen, Y. Yang, Y.-H. Chen, M. Qiu, R. J. Blaikie, B. Ding, J. Phys. Chem. C 2015, 119,18627

[49] A. Sobhani, A. Manjavacas, Y. Cao, M. J. McClain, F. J. García de Abajo, P. Nordlander, N. J. Halas, Nano Lett. 2015, 15, 6946

[50] J. Defrance, C. Lemaître, R. Ajib, J. Benedicto, E. Mallet, R. Pollès, J.-P. Plumey, M. Mihailovic, E. Centeno, C. Ciracì, D. Smith, A. Moreau, J. Op. Res. Soft. 2016, 4

[51] A. Moreau, C. Ciraci, D. R. Smith, Phys. Rev. B 2013, 87, 045401

[52] T. Ding, D. Sigle, L. Zhang, J. Mertens, B. de Nijs, J. Baumberg, ACS Nano, 2015, 9, 6110

[53] F. Benz, C. Tserkezis, L. O. Herrmann, B. de Nijs, A. Sanders, D. O. Sigle, L. Pukenas, S. D. Evans, J. Aizpurua, J. J. Baumberg, Nano Lett. 2015, 15, 669

[54] A. Moreau, C. Ciraci, J. J. Mock, R. T. Hill, Q. Wang, B. J. Wiley, A. Chilkoti, D. R. Smith, Nature 2012, 492, 86

[55] C. Ciracì, R. T. Hill, J. J. Mock, Y. Urzhumov, A. I. Fernández-Domínguez, S. A. Maier, J. B. Pendry, A. Chilkoti, D. R. Smith, Science 2012, 337, 1072

[56] J. Fedou, S. Viarbitskaya, R. Marty, J. Sharma, V. Paillard, E. Dujardin, A. Arbouet, Phys.Chem. Chem. Phys., 2013, 15, 4205 


\section{WILEY-VCH}

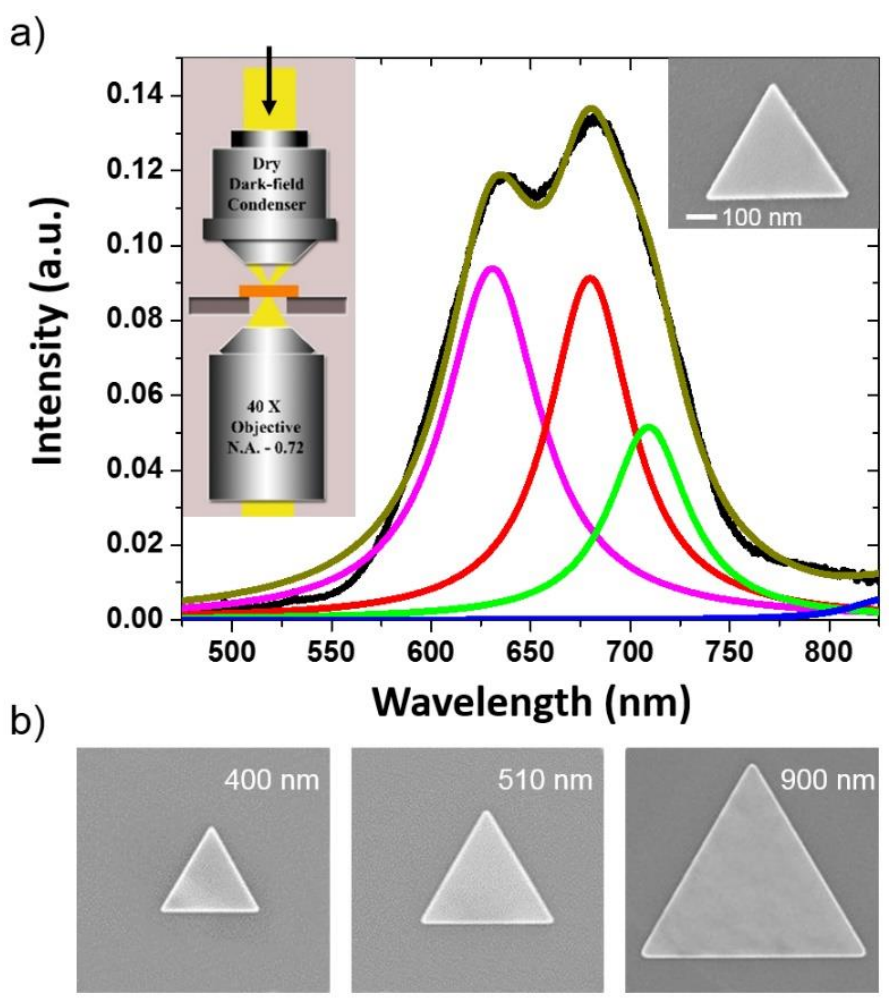

Figure 1: (a) DF scattering spectrum of a triangular gold nanoprism is shown in black (lateral dimension $460 \mathrm{~nm}$ and thickness is about $20 \mathrm{~nm}$ ). The pink, red, green and blue curves correspond to the Lorentzian decomposition done on the experimental spectrum. The curve in dark yellow color is the cumulative of the all the Lorentzian fitted peaks. Left inset: schematic diagram of the dark-field spectroscopy set up. White light is focused on the sample and the forward scattered light is collected in transmission. Right inset: SEM image of the sharp

triangular gold nanoprism deposited on ITO coated glass substrate from which the experimental spectrum is recorded (the scale bar in white is 100nm). (b) SEM images of three different prismatic cavities (side length is indicated is white).

a)

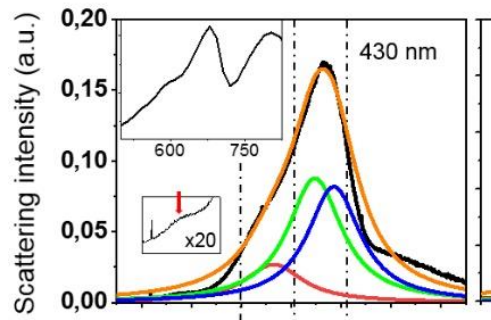

e)

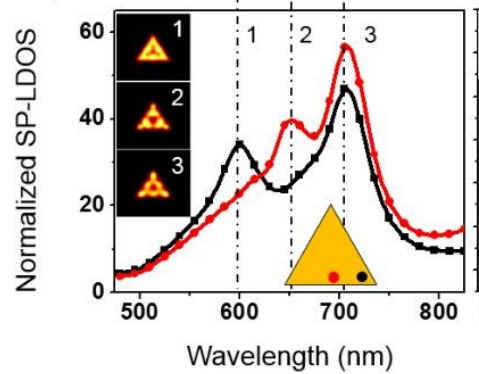

b)

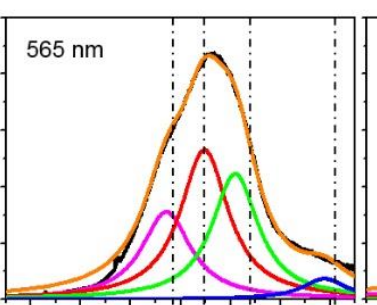

f)

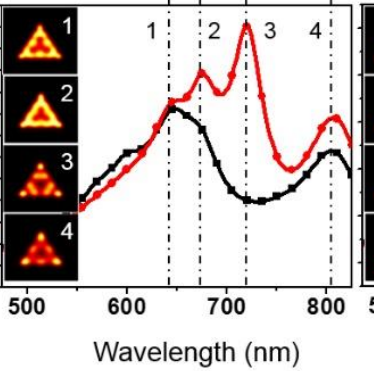

c)

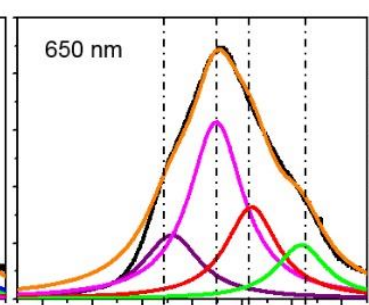

g)

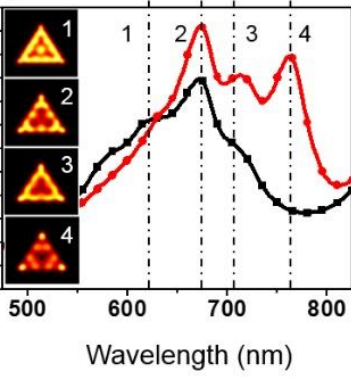

d)

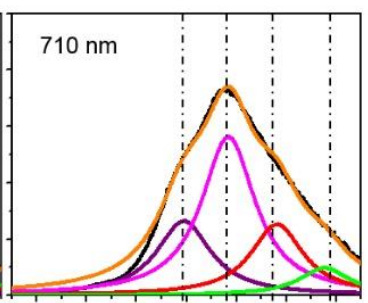

h)

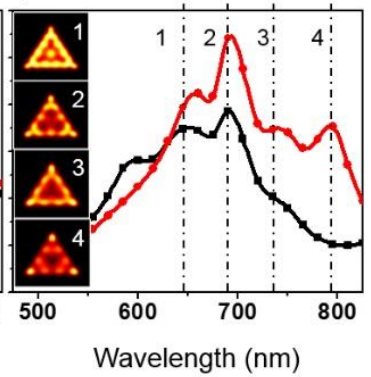




\section{WILEY-VCH}

Figure 2: (a-d) Experimental dark-field scattering spectra (black curves) from crystalline gold triangular cavities of size (a) $430 \mathrm{~nm}$ (Upper inset: simulated scattering cross-section. Lower inset: contribution of the transverse mode), (b) $565 \mathrm{~nm}$, (c) $650 \mathrm{~nm}$ and (d) $710 \mathrm{~nm}$ respectively. The spectra are fitted with Lorentzian peaks (colored peaks). The orange curves are the sum of the individual Lorentzian peaks. Corresponding near-field SPLDOS spectra calculated at one apex (in black) and middle of the edge (in red) of the nanoprisms are shown in (f) - (h). These positions are indicated by a red and a black dot on the schematic cavity in the inset in (f). The SP-LDOS maps corresponding to the main peaks are displayed in inset for each spectrum.

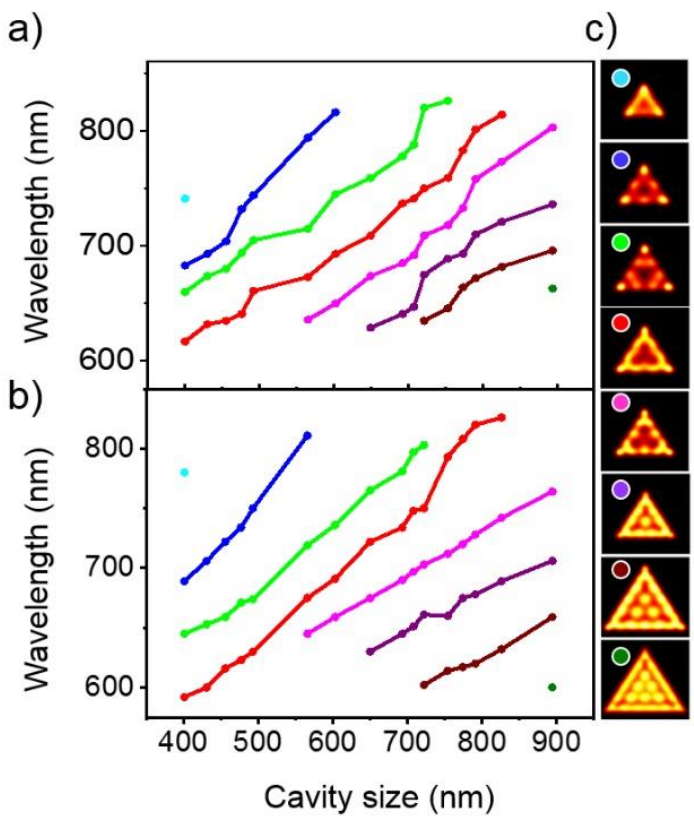

Figure 3: (a-b) High order SP resonance dispersion. The spectral position of the fitted (a) and computed (b) resonances is plotted as a function of the cavity edge size. A similar color code is used in Figure 2 and 3. (c) SP-LDOS maps corresponding to the resonances with the same color code.
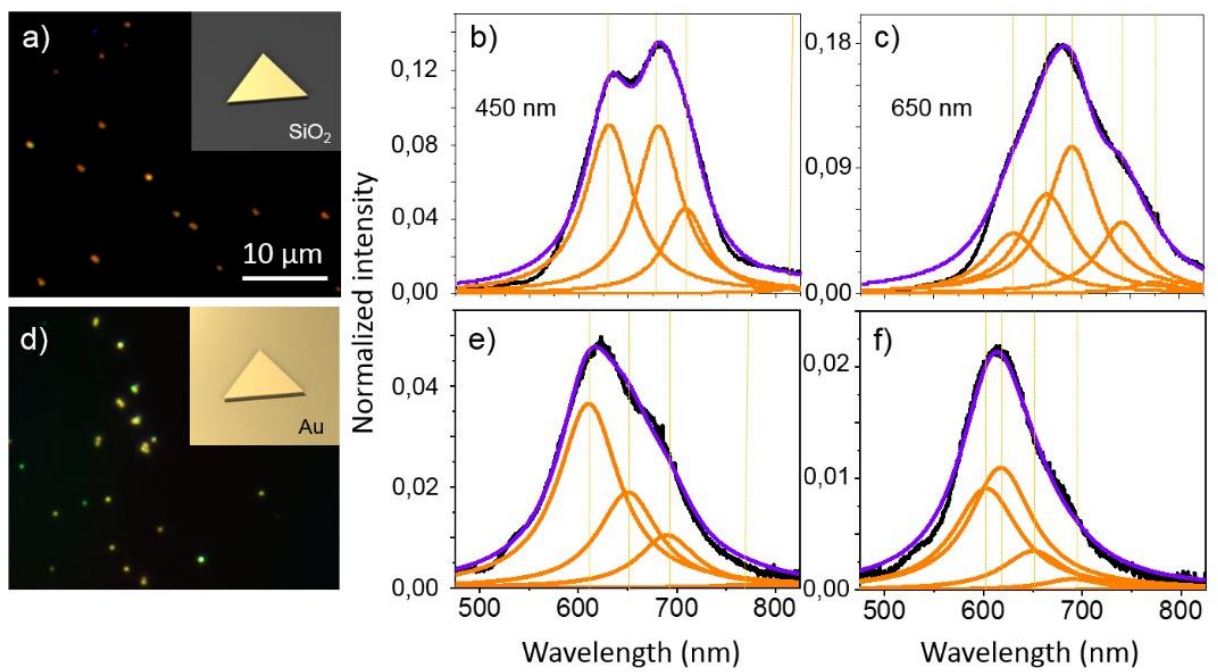

Figure 4: (a) and (d) show dark field images of Au cavities on ITO-covered glass and Au thin film respectively. Artistic views of the two configurations are shown in inset. DF spectra 


\section{WILEY-VCH}

(black curves) of Au cavities on an ITO-covered glass substrate with lateral sizes of (b) $460 \mathrm{~nm}$ and (c) $650 \mathrm{~nm}$ fitted by Lorentzian peaks (orange curves). The total fit (sum of the Lorentzian peaks) is displayed as a purple curve. (e)-(f) Similar as (b)-(c) for (e) $460 \mathrm{~nm}$ and (f) $650 \mathrm{~nm}$ Au cavities on a $30 \mathrm{~nm}$ thick Au film.
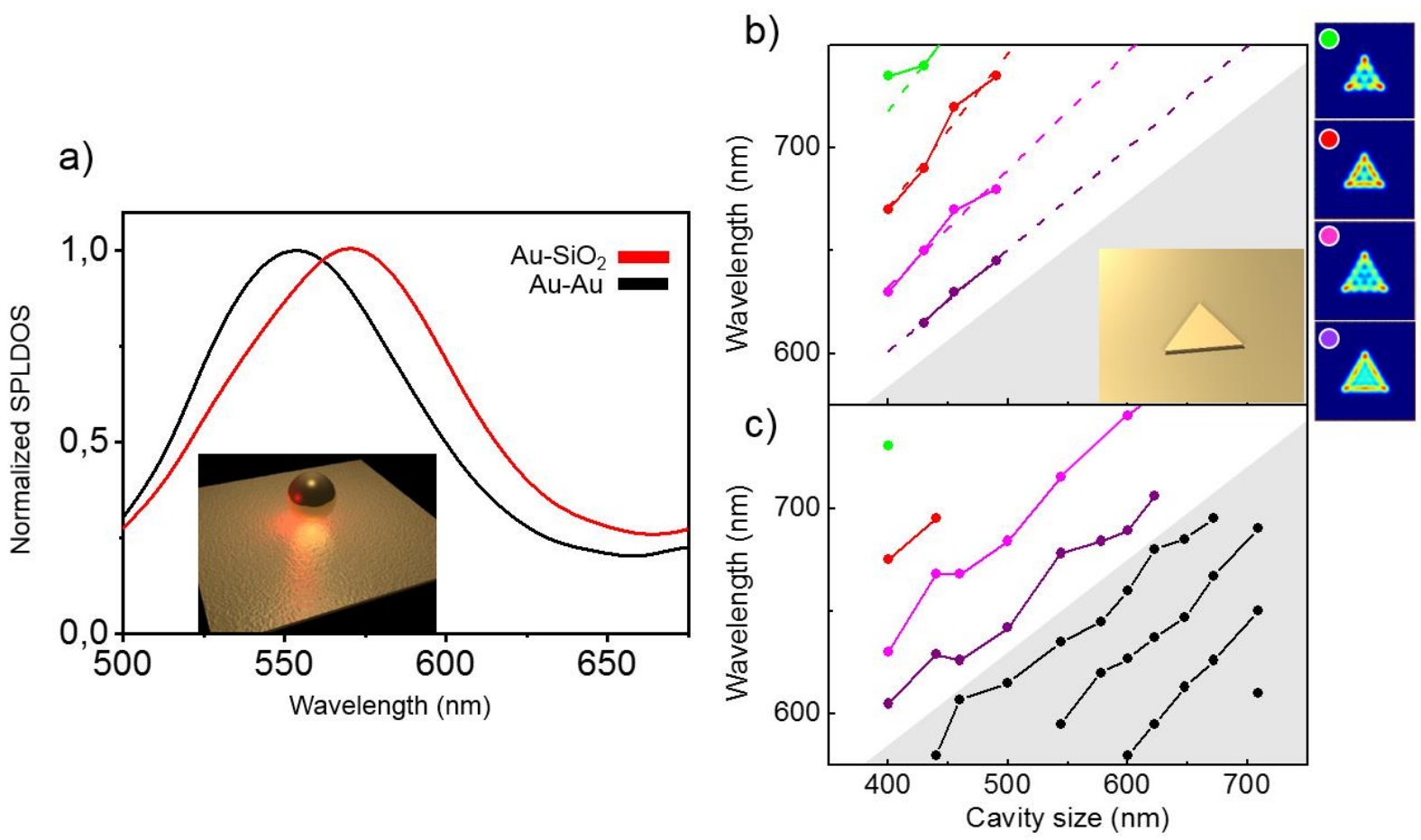

Figure 5: (a) Simulated SP-LDOS spectra of a $60 \mathrm{~nm}$ Au nanosphere positioned $10 \mathrm{~nm}$ above a $45 \mathrm{~nm}$ thick gold film. (b-c) High order SP resonance dispersion. The spectral position of the computed (a) and fitted (b) resonances is plotted as a function of the cavity edge size. A similar color code is used in Figure 2 and 3. SP-LDOS maps corresponding to the resonances with the same color code (colored dots).

a)

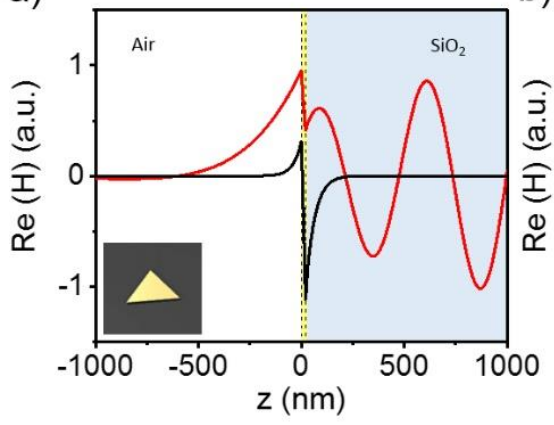

b)

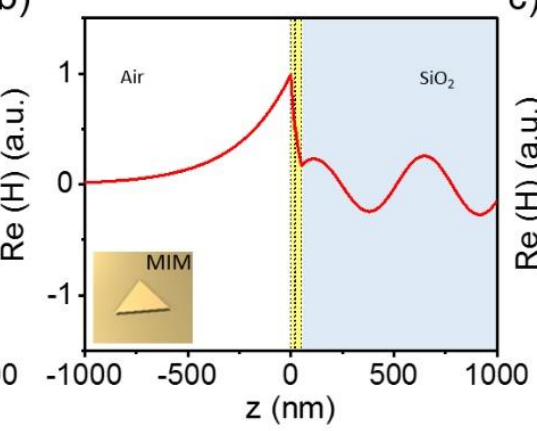

c)

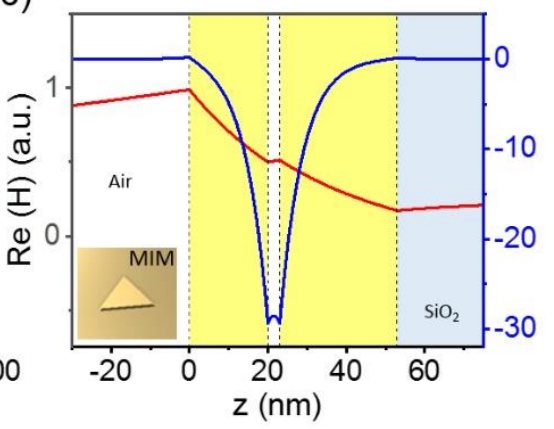

Figure 6: (a) Simulated profiles of the real part of the magnetic field associated to the plasmon bound to the upper air-Au (red curve) and lower Au-glass (black curve) interfaces at $\lambda=600$ $\mathrm{nm}$ on a $400 \mathrm{~nm}$ wide prism. (b) Similar as (a) for the upper plasmon in the MIM configuration. The growing oscillations of the field signal that the mode, computed by solving the dispersion relation for the structure, is leaking in the substrate. (c) Profiles of the real part

of the magnetic field of the upper plasmon and longitudinal gap plasmon in the MIM configuration. 

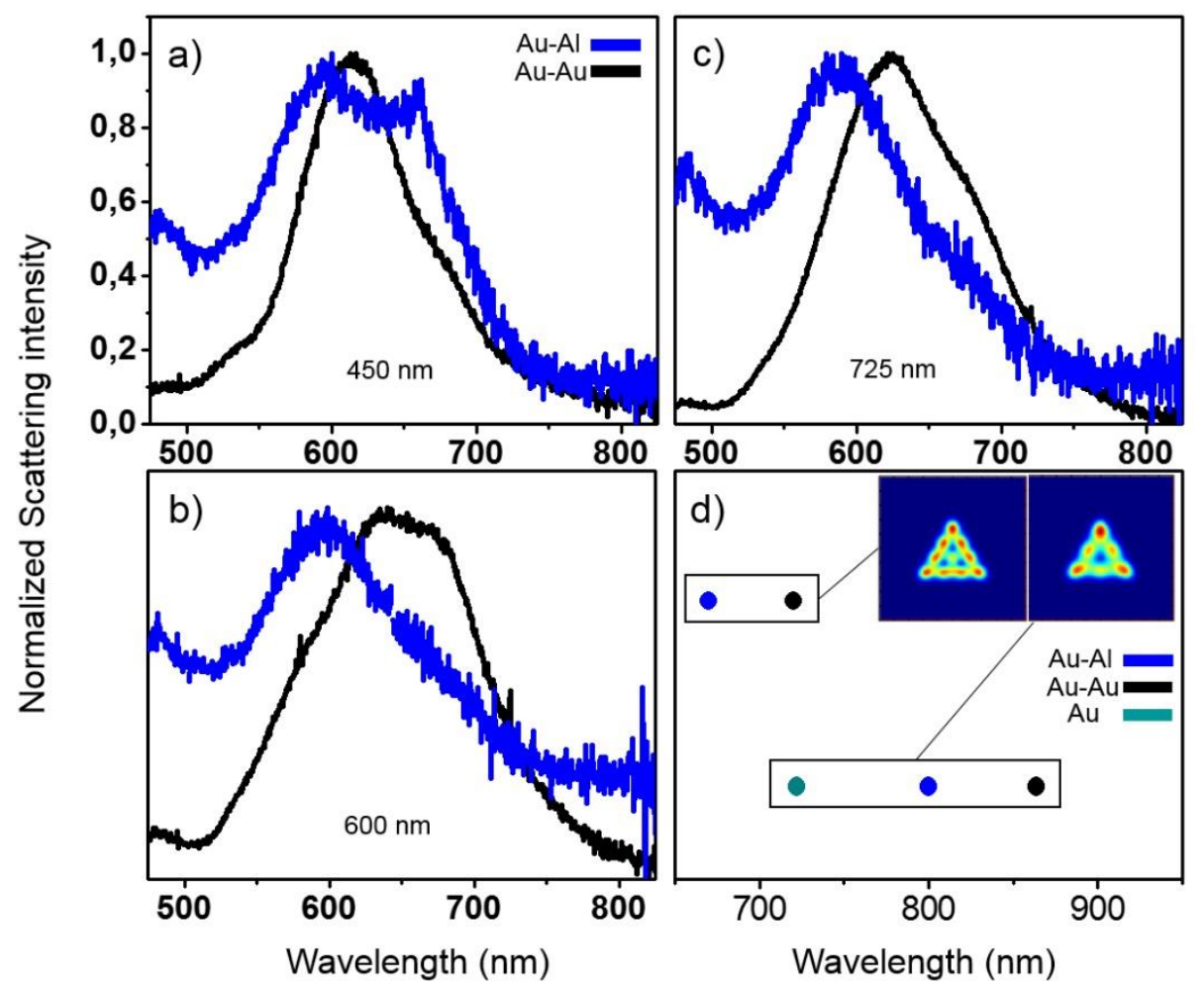

Figure 7: (a-c) Normalized DF scattering spectra of gold nanoprism of sizes (a) $450 \mathrm{~nm}$, (b) $600 \mathrm{~nm}$ and (c) $725 \mathrm{~nm}$ on two different metallic substrates $-30 \mathrm{~nm}$ thick gold film (black) and $30 \mathrm{~nm}$ thich aluminum film (blue). (d) Spectral position of two resonances as a function of the substrate nature (blue: aluminum; black: gold; cyan: ITO/glass). The spatial distributions corresponding to the two resonances are shown in inset. 


\section{WILEY-VCH}

The table of contents entry should be 50-60 words long, and the first phrase should be bold.

The spectral control of high order plasmon resonances on two-dimensional crystalline cavities is demonstrated in metal-insulator-metal geometries. The far-field optical signature and the dispersion of each single resonance is obtained through an original comparison with the computed plasmonic density of states. This work provides an innovative all-optical control of the blue- or red-shift of resonances with complex spatial distributions.

\section{Keyword}

\section{high-order plasmon resonances in MIM nanocavity}

U. Kumar 1, A. Cuche 1, J. Sharma 2, A. Moreau 3, G. Colas des Francs 4, C. Girard 1, E. Dujardin 1

Spectral tuning of high order plasmonic resonances in multimodal film-coupled crystalline cavities

\section{ToC figure}

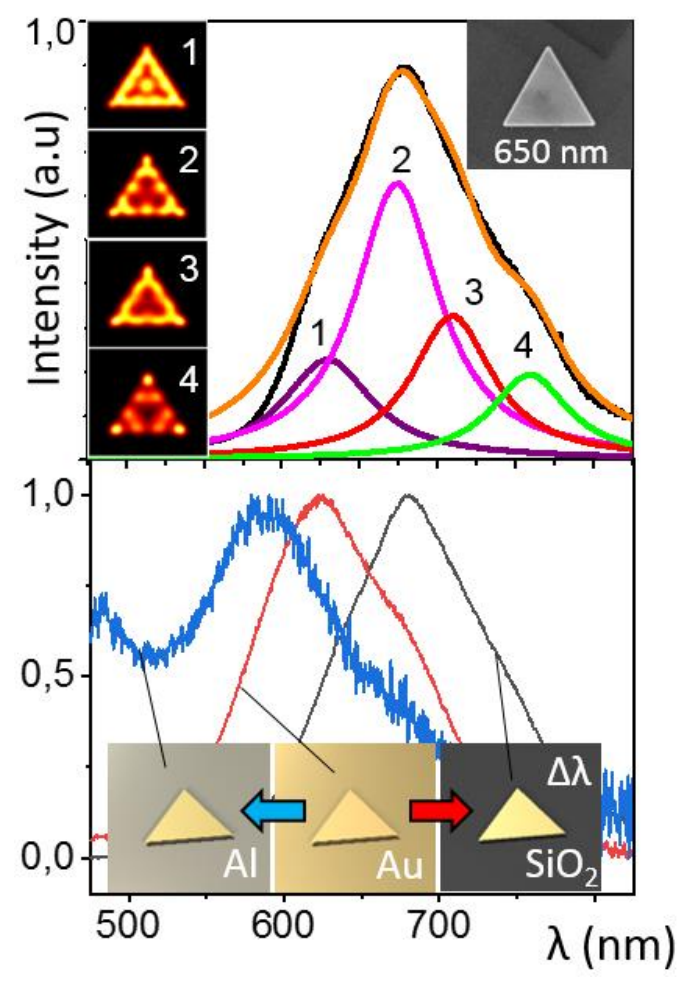

